\title{
Optimal control of COVID-19
}

\author{
Nacima Moussouni $^{a}$ and Mohamed Aliane ${ }^{b^{*}}$ \\ ${ }^{a}$ Laboratory L2CSP, University Mouloud Mammeri of Tizi Ouzou, Tizi-Ouzou, Algeria \\ ${ }^{b}$ Laboratory of Pure and Applied Mathematics, University Amar Telidji of Laghouat, Laghouat, Algeria \\ nmoussouni@yahoo.fr,m.aliane@lagh-univ.dz
}

\section{ARTICLE INFO}

Article History:

Received 21 April 2020

Accepted 10 September 2020

Available 30 January 2021

Keywords:

COVID-19

Epidemic

Optimal control

Shooting method

Euler discretization method

AMS Classification 2010:

$49 M 37$

\section{ABSTRACT}

Coronavirus disease of 2019 or COVID-19 (acronym for coronavirus disease 2019 ) is an emerging infectious disease caused by a strain of coronavirus called SARS-CoV-22, contagious with human-to-human transmission via respiratory droplets or by touching contaminated surfaces then touching them face. Faced with what the world lives, to define this problem, we have modeled it as an optimal control problem based on the models of William Ogilvy Kermack et Anderson Gray McKendrick, called SEIR model, modified by adding compartments suitable for our study. Our objective in this work is to maximize the number of recovered people while minimizing the number of infected. We solved the problem theoretically using the Pontryagin maximum principle, numerically we used and compared results of two methods namely the indirect method (shooting method) and the Euler discretization method, implemented in MATLAB.

\section{Introduction}

COVID-19 disease, which appeared in China in late 2019, is caused by SARS-CoV-2, a virus that belongs to the large family of coronaviruses. Very common, they can cause a simple cold as well as a serious respiratory infection like pneumonia, causing fatal epidemics as it was the case with Sras or Mers and this year, COVID-19. This virus passes into humans via animal secretions, under special conditions, then it is transmitted from human to human. Long-term contact is required for the transmission of this virus.

As with many infectious diseases, people with pre-existing chronic diseases such as hypertension, cardiovascular disease, diabetes, liver disease, respiratory disease, etc. Seem more likely to develop severe forms. Note that there are serious forms of the disease, even in young adults. Children under the age of fifteen are unlikely to trigger a severe form of coronavirus[CSSE].

In our work, we used a compartmental model in epidemiology based on the work of Kermack and Mckendrick [1, using ordinary differential equations we modeled this epidemic as an optimal control problem, our objective (and of course the objective of all humanity) is to maximize the number of recovered and minimize the number of infected and then draw the attention of the Algerian people to the seriousness of the situation. Initially the two authors proposed a founding model in which the population was divided between individuals susceptible to contracting the disease (compartment $S$ ), and infectious individuals (compartment $I$ ), it is noted model $S I$. Then they added the proportion of healed people, we get the SIR model [2].

In the first part of this work, we consider the SIR model with adding other compartments namely the proportion of individuals most exposed to the disease (chronic diseases and the elderly), people who have recovered as well as those who have died. In this model, compartment $S$ is necessary, since it must initially exist individuals who have not yet been infected, when an individual

*Corresponding Author 
in compartment $S$ is exposed to the disease, he does not necessarily become capable of transmitting it immediately, the virus requires a few days to make the individual sick [3, this is called the latency period, where the existence of compartment $\mathrm{E}$ then compartment $I$. After an individual has been infected, two scenarios can occur. First, the individual can die, in which case he belongs to compartment $D$, or recovered, in this case he belongs to compartment $R$. In our work, the command $u($.$) represents the vaccination rate [4]. Our$ goal is to minimize the number of infected people and maximize recoveries. The study is made in the case of Algeria. We estimate the states at 70 days and 150 days, from 02 April 2020 when the number of infected reached 986 people. This work is structured as follows: after a brief introduction, in section 2 , we defined the model used and explained the importance of each of its compartments. Section 3 is devoted to the theoretical resolution of the modeled problem, using the Pontryagin maximum principle [4-8]. The numerical resolution is evoked in section 4, we solved the considered problem with two numerical methods, namely the direct method (Euler discretization ) and an indirect method (shooting method). We developed an implementations with the MATLAB programming language, then we presented some simulation results. In the numerical application, two scenarios were considered, $T=70$ days and $T=150$ days, with and without an effective vaccine. In the rest of the work, we considered compartment $Q$ of people quarantined, the obtained problem is solved thoerically then numerically in section 5 . In the rest of the work, in the absence of treatment, the only remedy to face this epidemic is quarantine. We discussed and commented the results found with two methods of the previous section, in section 6 . Then we finished our document with a conclusion.

\section{Statement of the problem}

Starting from the basic model $(S I R)$, we tried to improve the equations so that it better correspond to reality. Indeed, many parameters come into play during the spread of a virus and are not taken into account in the basic model. The letter used to represent a compartment is also used to represent the number of individuals in the compartment. At all epidemic, some susceptible peoples become infected. The study is done over a period of $T=70$ days, and $T=150$ days. For $t \in[0, T]$, denote $\alpha$ the infection rate, $S($.$) verify$ the following relation:

$$
\dot{S}(t)=-\alpha S(t) I(t), S(0)=S_{0}>0 .
$$

As in any epidemic, infected people are vaccinated, note $u(t), t \in[0, T]$ the vaccination rate, $0 \leq u(.) \leq u_{\max }$, in this case we will have:

$$
\dot{S}(t)=-(\alpha I(t)+u(t)) S(t), S(0)=S_{0}>0 .
$$

$I($.$) Is such that:$

$$
\dot{I}(t)=\beta E(t)-(\delta+\gamma) I(t), I(0)=I_{0}>0,
$$

where $E(),. t \in[0, T]$ denotes people witch are exposed to the epidemic, it verify the following differential equation:

$$
\dot{E}(t)=\alpha I(t) S(t)-\beta E(t), E(0)=E_{0}>0 .
$$

As in any epidemic, infected people are either recovered (compartment $R$ ) after being vaccinated or dead (compartment $D$ ), this is governed by the following differential equations:

$$
\dot{R}(t)=\gamma I(t)+u(t) S(t), \quad R(0)=R_{0},
$$

and

$$
\dot{D}(t)=\delta I(t), \quad D(0)=D_{0}, t \in[0, T] .
$$

The positive coefficients are such that:

$\alpha>0$ is infection rate, incubation rate $\beta$, is the rate of latent individuals becoming infectious. Given the known average duration of incubation $Y, \beta=\frac{1}{Y}, \gamma=\frac{1}{K}$, is determined by the average duration of recovery $K$, of infection. $\delta$ is the mortality rate.

The evolution of the considered states is shown in the following figure(see Figure 1):

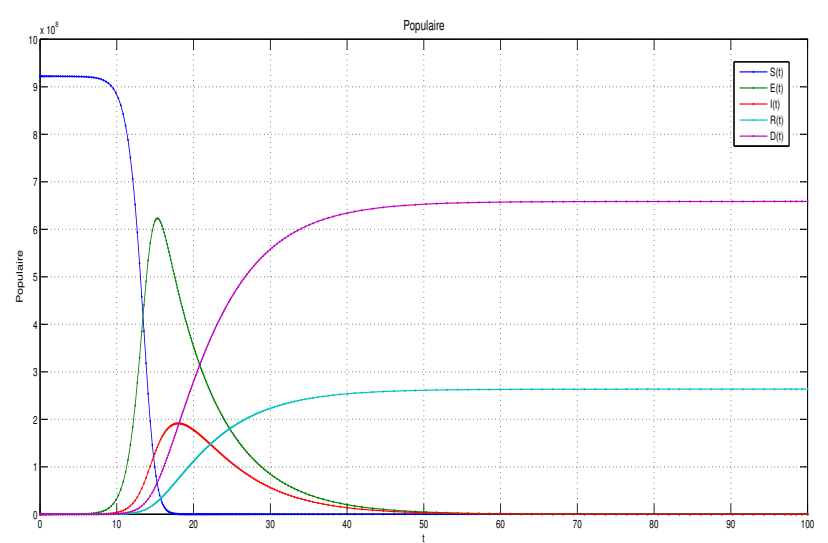

Figure 1. Evolution of stats.

After this modeling, our goal is to minimize the number of infected people and maximize recoveries. The objective function in our case is:

$$
J(u)=-I(T)+R(T) \rightarrow \max _{u} .
$$


We will therefore solve a following optimal control problem:

$$
\begin{cases}J(u)=-I(T)+R(T) \rightarrow \max _{u}, \\ \dot{S}(t)=(-\alpha I(t)-u(t)) S(t), & S(0)=S_{0}>0, \\ \dot{E}(t)=\alpha I(t) S(t)-\beta E(t), & E(0)=E_{0}>0, \\ \dot{I}(t)=\beta E(t)-(\delta+\gamma) I(t), & I(0)=I_{0}>0, \\ \dot{R}(t)=\gamma I(t)+u(t) S(t), & R(0)=R_{0}>0, \\ \dot{D}(t)=\delta I(t), & D(0)=D_{0}>0, \\ 0 \leq u(t) \leq u_{\max }, t \in[0, T] . & \end{cases}
$$

\section{Theoretical resolution}

Taking the case of Algeria, out of 10.0000 healthy people, 3000 are exposed to the virus (among others doctors, nurses and people at risk). As of 02 April 2020, 986 person are infected in this country, 83 died and 108 were cured(Pasteur Institute results).

Each exposed person $(E)$ is transformed into the infected person $(I)$ with rate $\beta$, the incubation period refer to the time of infection to clinical symptoms of the disease. We shall assume that incubation period is a maximum of 7 days then $\beta=\frac{1}{7}$. An infected person is healed up after 6 days, as well $\gamma=\frac{1}{6}[3]$.

This obtained problem is solved theoretically by using the Pontryagin maximum principle(PMP), then numerically, we implemented two methods on MATLAB namely the indirect method (shooting method) and a direct one.

The Hamiltonian of problem (7) is given for $t \in$ $[0, T]$ as follows:

$$
\begin{aligned}
H(x(t), p(t), u(t))= & -p_{S}(t) S(t)(\alpha I(t)+u(t)) \\
& +p_{E}(t)(\alpha I(t) S(t)-\beta E(t)) \\
& +p_{I}(t)(\beta E(t)-(\delta+\gamma) I(t)) \\
& +p_{R}(t)(\gamma I(t)+u(t) S(t)) \\
& +p_{D}(t)(\delta I(t)),
\end{aligned}
$$

where, $x(t)=(S(t), I(t), E(t), R(t), D(t))$ and $p(t)=\left(p_{S}(t), p_{I}(t), p_{E}(t), p_{R}(t), p_{D}(t)\right)$ is adjoint vector, it is solution of the system:

$$
\left\{\begin{aligned}
\dot{p}_{S}(t)= & \alpha I(t)\left(p_{S}(t)-p_{E}(t)\right) \\
& -\left(-p_{S}(t)+p_{R}(t)\right) u(t) \\
\dot{p}_{E}(t)= & \beta\left(p_{I}(t)-p_{E}(t)\right) \\
\dot{p}_{I}(t)= & \alpha S(t)\left(p_{S}(t)-p_{E}(t)\right)+(\delta+\gamma) p_{I}(t) \\
& -\gamma p_{R}(t)-\delta p_{D}(t) \\
\dot{p}_{R}(t)= & 0 \\
\dot{p}_{D}(t)= & 0, t \in[0, T]
\end{aligned}\right.
$$

The transversality conditions are such that:

$$
\begin{aligned}
& p_{S}(T)=-\frac{\partial g(S(T), E(T), I(T), R(T), D(T))}{\partial S(T)}, \\
& p_{E}(T)=-\frac{\partial g(S(T), E(T), I(T), R(T)), D(T)}{\partial E(T)}, \\
& p_{I}(T)=-\frac{\partial g(S(T), E(T), I(T), R(T), D(T))}{\partial I(T)}, \\
& p_{R}(T)=-\frac{\partial g(S(T), E(T), I(T), R(T), D(T))}{\partial R(T)}, \\
& p_{D}(T)=-\frac{\partial g(S(T), E(T), I(T), R(T), D(T))}{\partial D(T)} .
\end{aligned}
$$

where

$g(S(T), E(T), I(T), R(T), D(T))=-I(T)+R(T)$.

That gives:

$$
\begin{gathered}
p_{S}(T)=0, p_{E}(T)=0, p_{I}(T)=-1, \\
p_{R}(T)=1, p_{D}(T)=0 .
\end{gathered}
$$

Let us Calculate the optimal controle $u($.$) :$

The Hamiltonien's maximum is given by:

$$
\begin{aligned}
H^{*}= & \max _{0 \leq u(t) \leq u_{\max }} H(t, x(t), p(t), u(t)) \\
= & -\alpha S(t) I(t)\left(p_{S}(t)-p_{E}(t)\right)-\beta p_{E}(t) E(t) \\
& +p_{I}(t)(\beta E(t)-(\delta+\gamma) I(t))+\gamma p_{R}(t) I(t) \\
& +\delta p_{D}(t) I(t) \\
& +S(t) \max _{0 \leq u(t) \leq u_{\max }}\left[\left(-p_{S}(t)+p_{R}(t)\right) u(t)\right]
\end{aligned}
$$

where $H^{*}=H\left(x^{*}(t), p^{*}(t), u^{*}(t)\right)$.

The control which maximizes the Hamiltonian is

$$
u^{*}(t)= \begin{cases}u_{\max } & \text { if } p_{S}(t) \leq 1 \\ 0 & \text { if } p_{S}(t)>1\end{cases}
$$

Then:

$$
u^{*}(t)=-u_{\max } \min \left(0, \operatorname{sign}\left(p_{S}(t)-1\right)\right) .
$$




\section{Numerical implementation and methods}

To compute the solution of the optimality system and maximize the objective functional $J(u)$, two implementations are considered: indirect and direct methods, these implementations were compared in terms of model dynamics for the first 70 days $(t \in[0,70])$ using both the parameter values $\alpha, \beta, \gamma$, and $\sigma$ in section 3 , and the initial conditions

$$
\left\{\begin{array}{c}
S(0)=10^{6}, \\
E(0)=3000, \\
I(0)=986, \\
R(0)=103, \\
D(0)=86 .
\end{array}\right.
$$

Indirect method to solve optimal control problems are based on the Pontryagin's maximum principle to compute the optimal solution. Therefor, these methods require the state system with initial value [4, and necessary conditions [4,5,7], to find the solution of a given optimal control problem, by convering it into a boundary value problem.

On the other hand, direct methods use discretization with respect to time to solve optimal control problems, in which the cost functionl is directly optimized, treating the optimal control problem as a nonlinear optimization problem $(N L P)$.

Some advantages and drawbacks can be pointed out to both indirect and direct methods. According to Trélat [4], direct methods are more robust and less sensitive to the choice of the initial conditions than indirect methods, being more easier to implement. However, in comparison with indirect methods, reaching to a desirable precision is not so easy when direct methods are employed. In addition, the author states not only the possibility of obtain local minima when the direct discretization of an optimal control problem is employed, but also the necessity of a large amount of memory, which in turn can lead to inefficiencies when, for instance, a large dimension problem is considered. On the other hand, indirect methods provide high levels of numerical accuracy, but their implementation can be quite difficult due to the necessity of computing derivatives and necessary conditions related to the PMP.

\subsection{Resolution by Shooting method}

The shooting method is based on the Pontryagin's maximum principle [9]. It consists in finding a zero of the shooting function associated with the original problem. It is a fast, high-precision method that does not require assumptions about the control structure. The shooting method consists in three main steps [7, 8]:

- Step 1: Form a boundary value problem using the model equations and the adjoint vectors equations as well as the transversality conditions.

- Step 2: Determine the shooting function.

- Step 3: Solve a system of nonlinear equations.

The Pontryagin maximum principle leads us to the following boundary value problem:

$$
\left\{\begin{aligned}
\dot{x}_{1}(t)=-\alpha x_{3}(t) x_{1}(t)+F\left(p_{1}(t)\right) x_{1}(t), \\
\dot{x}_{2}(t)=\alpha x_{3}(t) x_{1}(t)-\beta x_{2}(t), \\
\dot{x}_{3}(t)=\beta x_{2}(t)-(\sigma+\gamma) x_{3}(t), \\
\dot{x}_{4}(t)=\gamma x_{3}(t)-F\left(p_{1}(t)\right) x_{1}(t), \\
\dot{x}_{5}(t)=\sigma x_{3}(t), \\
\dot{p}_{1}(t)=\alpha x_{3}(t)\left(p_{1}(t)-p_{2}(t)\right) \\
\quad-\left(p_{1}(t)-p_{4}(t)\right) F\left(p_{1}(t)\right), \\
\dot{p}_{2}(t)=\beta\left(p_{2}(t)-p_{3}(t)\right), \\
\dot{p}_{3}(t)=\alpha x_{1}(t)\left(p_{1}(t)-p_{2}(t)\right)+(\sigma+\gamma) p_{3}(t), \\
\quad-\gamma p_{4}(t)-\sigma p_{5}(t), \\
\dot{p}_{4}(t)=0, \\
\dot{p}_{5}(t)=0, \\
x_{1}(0)=S_{0}, x_{2}(0)=E_{0}, x_{3}(0)=I_{0}, \\
x_{4}(0)=R_{0}, x_{5}(0)=D(0), p_{1}(T)=p_{2}(T)=0, \\
p_{3}(T)=-1, p_{4}(T)=1, p_{5}(T)=0, t \in[0, T] .
\end{aligned}\right.
$$

where

$$
\begin{aligned}
x(t) & =\left(x_{1}(t), x_{2}(t), x_{3}(t), x_{4}(t), x_{5}(t)\right) \\
& =(S(t), E(t), I(t), R(t), D(t)), \\
p(t) & =\left(p_{j}(t), j=1 \ldots 5\right) \\
& =\left(p_{S}(t), p_{E}(t), p_{I}(t), p_{R}(t), p_{D}(t)\right) .
\end{aligned}
$$

and

$$
F\left(p_{1}(t)\right)=u_{\max } \min \left(0, \operatorname{sign}\left(p_{1}(t)-1\right) .\right.
$$

We construct the following shooting function:

$$
\begin{aligned}
& G: R^{10} \quad \longrightarrow R^{10} \\
& (p(0), p(T)) \longmapsto G(p(0), p(T)) \text {, }
\end{aligned}
$$




$$
G(p(0), p(T))=\left(\begin{array}{c}
p_{1}(0)-\lambda_{1} \\
p_{2}(0)-\lambda_{2} \\
p_{3}(0)-\lambda_{3} \\
p_{4}(0)-1 \\
p_{5}(0) \\
p_{1}(T) \\
p_{2}(T) \\
p_{3}(T)+1 \\
p_{4}(T)-1 \\
p_{5}(T)
\end{array}\right)
$$

where $\lambda_{i} \in R, i=1 \ldots 3$.

Hence, the problem (11) is equivalent to the following problem:

$$
\left\{\begin{array}{l}
\dot{z}_{1}(t)=-\alpha z_{3}(t) z_{1}(t)+F\left(z_{6}(t)\right) z_{1}(t) \\
\dot{z}_{2}(t)=\alpha z_{3}(t) x_{1}(t)-\beta z_{2}(t), \\
\dot{z}_{3}(t)=\beta z_{2}(t)-(\sigma+\gamma) z_{3}(t), \\
\dot{z}_{4}(t)=-F\left(z_{6}(t)\right) z_{1}(t)+\gamma z_{3}(t) \\
\dot{z}_{5}(t)=\sigma z_{3}(t) \\
\dot{z}_{6}(t)=\alpha z_{3}(t)\left(z_{6}(t)-z_{7}(t)\right) \\
\quad-\left(z_{6}(t)-z_{9}(t)\right)+F\left(z_{6}(t)\right) \\
\dot{z}_{7}(t)=\beta\left(z_{7}(t)-z_{8}(t)\right) \\
\dot{z}_{8}(t)=\alpha z_{1}(t)\left(z_{6}(t)-z_{7}(t)\right)+(\sigma+\gamma) z_{8}(t) \\
\quad-\gamma z_{9}(t)-\sigma z_{10}(t), \\
\dot{z}_{9}(t)=0 \\
\dot{z}_{10}(t)=0 \\
z_{1}(0)=S_{0}, z_{2}(0)=E_{0}, z_{3}(0)=I_{0}, z_{4}(0)=R_{0}, \\
z_{5}(0)=D_{0}, G(p(0), p(T))=0, t \in[0, T],
\end{array}\right.
$$

where

$$
z(t)=\left(z_{j}(t), j=1, \ldots, 10\right)=(x(t), p(t))
$$

and

$$
F\left(z_{6}(t)\right)=u_{\max } \min \left(0, \operatorname{sign}\left(z_{6}(t)-1\right) .\right.
$$

Using shooting method, in the presence of an effective vaccine, after 70 days, the results found are illustrated in the following figure (see Figure2):
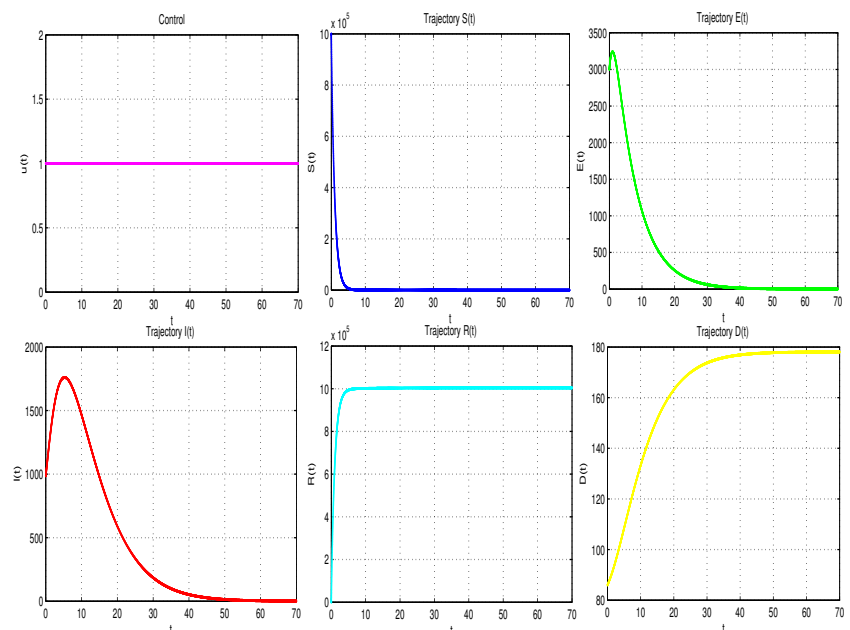

Figure 2. Evolution of stats after 70 days with vaccine.

After 70 days, in the presence of an effective treatment, the number of people recovered is very high, the number of infected decreases considerably.

In the absence of an effective vaccine, we will use the shooting method, after for $T=150$ days, the results are illustrate in the following figure(see Figur(3):
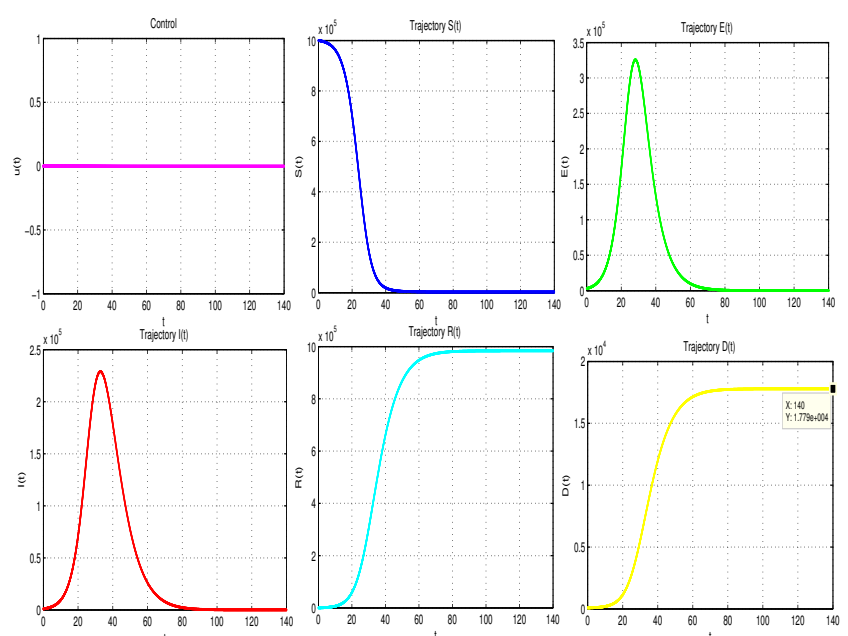

Figure 3. Evolution of stats after 150 days without vaccine.

After 150 days, in the absence of an effective vaccine, the number of susceptible individuals does not almost decrease, the number of deaths increases. The number of susceptible individuals as well as the number of exposed to the virus increases considerably(see Figure 3). The execution time of the shooting method is $C P U$ time equals $3.18 s$. 


\subsection{Resolution by the Euler discretization method}

For a number of subintervals $N$ chosen in advance, we will have the step of discretization $h=\frac{T}{N}$ and the following times:

$$
0=t_{0}<t_{1}<\cdots<t_{N-1}<t_{N}=T .
$$

The application of the Euler discretization scheme for solving boundary value problems gives us the following nonlinear programming problem:

$$
\begin{aligned}
& \text { Minimize } J(u)=I(T)-R(T), \\
& S\left(t_{k+1}\right)=S\left(t_{k}\right)-h\left(\alpha I\left(t_{k}\right)+u\left(t_{k}\right)\right) S\left(t_{k}\right), \\
& E\left(t_{k+1}\right)=E\left(t_{k}\right)+h\left(\alpha I\left(t_{k}\right) S\left(t_{k}\right)-\beta E\left(t_{k}\right)\right), \\
& I\left(t_{k+1}\right)=I\left(t_{k}\right)+h\left(\beta E\left(t_{k}\right)-(\sigma+\gamma) I\left(t_{k}\right)\right), \\
& R\left(t_{k+1}\right)=R\left(t_{k}\right)+h\left(\gamma I\left(t_{k}\right)+u\left(t_{k}\right) S\left(t_{k}\right)\right), \\
& D\left(t_{k+1}\right)=D\left(t_{k}\right)+h \sigma I\left(t_{k}\right), k=0,1, \ldots, N-1, \\
& S(0)=S_{0}, E(0)=E_{0}, I(0)=I_{0}, R(0)=0, \\
& D(0)=D_{0}, \quad 0 \leq u\left(t_{k}\right) \leq u_{\max }, \quad k=0,1, \ldots, N .
\end{aligned}
$$

We also solved the nonlinear program (14) with the interior-point method implemented in MATLAB for $N=1500$. The obtained results are presented in figure (see Figure4)
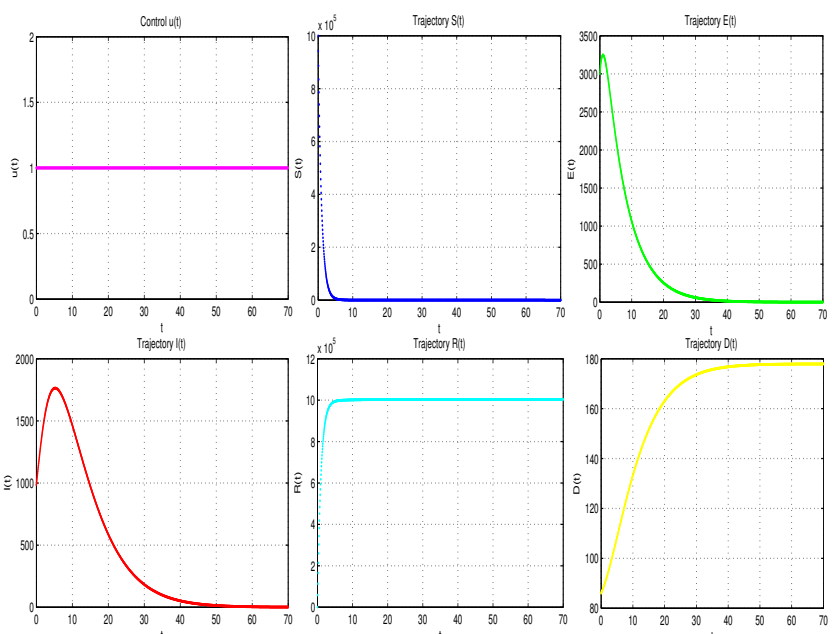

Figure 4. Evolution of stats with vaccine, after 70 days.

After 70 days, in the presence of an effective vaccine, the number of infected individuals decreases, and the number of deaths stabilizes around 180 dead from 40 days. The number of people recovered is considerable(see Figure 4).

It has been noted in the previous sections that in the absence of treatment the number of infected persons increases considerably, the best solution is therefore isolation. The execution time of the shooting method is $C P U$ time equals $26.85 \mathrm{~s}$.

\section{Advantage of quarantine}

Based on China's experience, the vast majority of people who contracted COVID-19 contracted it from contact with infected relatives, hence the importance of isolation so that the epidemic can be controlled. taking the example of the Ebola epidemic and the case of China with this new virus, peoples had been deprived of their freedom of movement, this has paid off, the number of infected individuals has decreased considerably.

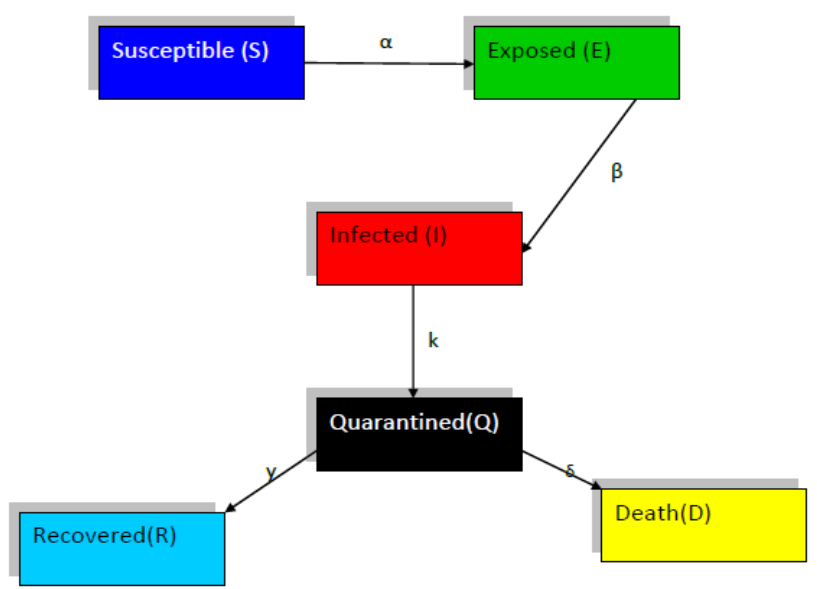

Figure 5. SEIQR model.

Based on this protection strategy, we modeled another problem inspired by the SEIRD model, by adding the compartment of quarantined people (see Figure 5), the following optimal control problem has been obtained:

$$
\left\{\begin{array}{l}
J(u)=-I(T)+R(T) \rightarrow \max _{u}, \\
\dot{S}(t)=-(\alpha I(t)+u(t)) S(t), \quad S(0)=S_{0}>0, \\
\dot{E}(t)=\alpha I(t) S(t)-\beta E(t), \quad E(0)=E_{0}>0, \\
\dot{I}(t)=\beta E(t)-\kappa I(t), I(0)=I_{0}>0, \\
\dot{Q}(t)=\kappa I(t)-(\gamma+\sigma) Q(t), Q(0)=Q_{0}>0, \\
\dot{R}(t)=\gamma Q(t)+u(t) S(t) \quad R(0)=R_{0}, \\
\dot{D}(t)=\sigma Q(t), \quad D(0)=D_{0}, \\
0 \leq u(t) \leq u_{\max }, t \in[0, T], T: \text { Fix. }
\end{array}\right.
$$

Where: $\alpha$ is the infection rate, $\beta^{-1}$ average latente time, $\kappa^{-1}$ average isolation time,$\gamma$ is cure time and $\sigma$ is the mortality rate.

We notice

$$
\dot{S}(t)+\dot{E}(t)+\dot{I}(t)+\dot{Q}(t)+\dot{R}(t)+\dot{D}(t)=0,
$$


therefore

$S(t)+E(t)+I(t)+Q(t)+R(t)+D(t)=N_{P}, N_{p} \in R$, and like

$$
S(0)+E(0)+I(0)+R(0)+D(0)=N_{p},
$$

deduce that

$$
N_{p}=1005075
$$

therefore

$$
D(t)=-S(t)-E(t)-I(t)-Q(t)-R(t)+N_{p} .
$$

We can simplify the problem.

$$
\left\{\begin{array}{l}
J(u)=-I(T)+R(T) \rightarrow \max _{u}, \\
\dot{S}(t)=-(\alpha I(t)+u(t)) S(t), \quad S(0)=S_{0}>0, \\
\dot{E}(t)=\alpha I(t) S(t)-\beta E(t), \quad E(0)=E_{0}>0, \\
\dot{I}(t)=\beta E(t)-\kappa I(t), \quad I(0)=I_{0}>0, \\
\dot{Q}(t)=\kappa I(t)-(\gamma+\sigma) Q(t), Q(0)=Q_{0}>0, \\
\dot{R}(t)=\gamma Q(t)+u(t) S(t), \quad R(0)=R_{0}, \\
0 \leq u(t) \leq u_{\max }, t \in[0, T], T: \text { Fix. }
\end{array}\right.
$$

\subsection{Theoretical resolution}

The Hamiltonian of problem (16) is given for $t \in[0, T]$ as follows:

$$
\begin{aligned}
H(x(t), p(t), u(t))= & p_{S}(t)(-\alpha I(t)-u(t)) S(t) \\
& +p_{E}(t)(\alpha I(t) S(t)-\beta E(t)) \\
& +p_{I}(t)(\beta E(t)-\kappa I(t)) \\
& +p_{Q}(t)(\kappa I(t)-(\gamma+\sigma) Q(t)) \\
& +p_{R}(t)(\gamma Q(t)+u(t) S(t)),
\end{aligned}
$$

where, $x(t)=(S(t), E(t), I(t), Q(t), R(t))$ and $p(t)=\left(p_{S}(t), p_{E}(t), p_{I}(t), p_{Q}(t), p_{R}(t)\right)$ is adjoint vector, it's solution of the system:

$$
\left\{\begin{aligned}
\dot{p}_{S}(t)=\alpha I(t)\left(p_{S}(t)-p_{E}(t)\right) & \\
& +\left(p_{S}(t)-p_{R}(t)\right) u(t), \\
\dot{p}_{E}(t) & =\beta\left(p_{E}(t)-p_{I}(t)\right), \\
\dot{p}_{I}(t) & =\alpha S(t)\left(p_{S}(t)-p_{E}(t)\right) \\
& +\kappa\left(p_{I}(t)-p_{Q}(t)\right), \\
\dot{p}_{Q}(t) & =(\gamma+\sigma) p_{Q}(t)-\gamma p_{R}(t), \\
\dot{p}_{R}(t) & =0, t \in[0, T] .
\end{aligned}\right.
$$

The transversality conditions are such that:

$$
\begin{aligned}
& p_{S}(T)=-\frac{\partial g(S(T), E(T), I(T), Q(T), R(T))}{\partial S(T)}, \\
& p_{E}(T)=-\frac{\partial g(S(T), E(T), Q(T), I(T), R(T))}{\partial E(T)} \\
& p_{I}(T)=-\frac{\partial g(S(T), E(T), Q(T), I(T), R(T))}{\partial I(T)} \\
& p_{Q}(T)=-\frac{\partial g(S(T), E(T), Q(T), I(T), R(T))}{\partial Q(T)}, \\
& p_{R}(T)=-\frac{\partial g(S(T), E(T), Q(T), I(T), R(T))}{\partial R(T)}
\end{aligned}
$$

That gives:

$$
\begin{gathered}
p_{S}(T)=0, p_{E}(T)=0, p_{I}(T)=-1, \\
p_{Q}(T)=0, p_{R}(T)=1 . \\
\dot{p}_{R}(t)=0, p_{R}(t)=1, \forall t \in[0, T],
\end{gathered}
$$

implies

$$
\begin{gathered}
p_{R}(t)=1, \forall t \in[0, T] . \\
\dot{p}_{Q}(t)=(\gamma+\sigma) p_{Q}(t)-\gamma p_{R}(t), \forall t \in[0, T], \\
p_{Q}(T)=0,
\end{gathered}
$$

implies

$$
p_{Q}(t)=e^{(\gamma+\sigma)(t-T)}+\frac{\gamma}{\gamma+\sigma}, t \in[0, T] .
$$

Let us Calculate the optimal controle $u($.$) :$

The Hamiltonien's maximum is given by

$$
\begin{aligned}
H^{*}= & \max _{0 \leq u(t) \leq u_{\max }} H(x(t), p(t), u(t)) \\
= & -\alpha p_{S}(t) S(t) I(t)+p_{E}(t)(\alpha I(t) S(t) \\
& -\beta E(t))+p_{I}(t)(\beta E(t)-\kappa I(t)) \\
& +p_{Q}(t)(\kappa I(t)-(\gamma+\sigma) Q(t)) \\
& +\gamma p_{R}(t) Q(t) \\
& +S(t) \max _{0 \leq u(t) \leq u_{\max }}\left[\left(-p_{S}(t)+p_{R}(t)\right) u(t)\right] .
\end{aligned}
$$

where $H^{*}=H\left(x^{*}(t), p^{*}(t), u^{*}(t)\right)$.

The control which maximizes the Hamiltonian is

$$
u^{*}(t)= \begin{cases}u_{\max } & \text { if } p_{S}(t) \leq 1 \\ 0 & \text { if } p_{S}(t)>1\end{cases}
$$

Then:

$$
u^{*}(t)=-u_{\max } \min \left(0, \operatorname{sign}\left(p_{S}(t)-1\right)\right) .
$$

\section{Numerical implementation}

To compute the solution of the optimality system and maximize the objective functional $J(u)$, two implementations are considered: indirect and direct methods, these implementations were compared in terms of model dynamics for the first 70 days $(t \in[0,70])$ using both the parameter values 
$\alpha, \beta, \gamma$, and $\sigma$ in section 3 , and the initial conditions:

$$
\left\{\begin{array}{c}
S(0)=10^{6}, \\
E(0)=3000, \\
I(0)=986, \\
Q(0)=900, \\
R(0)=103, \\
D(0)=86 .
\end{array}\right.
$$

The results obtained for $T=150$ days in the absence of the vaccine, using the shooting method are illustrated in the following figure(see Figure6)

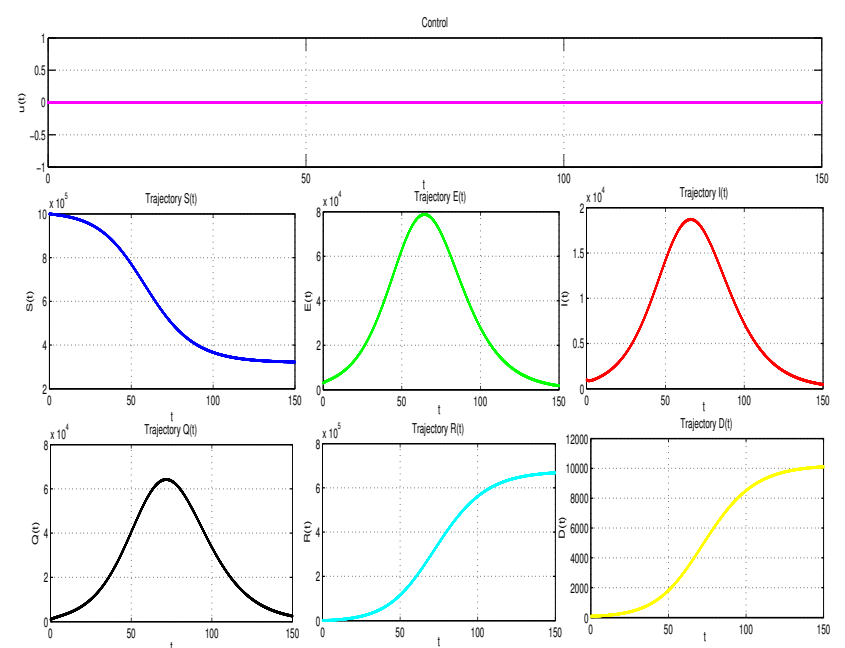

Figure 6. Evolution of stats without vaccine, after 150 days.

The results obtained for $T=70$ days in the absence of the vaccine, using the Euler discretization method are illustrated in the following figure(see Figure7):

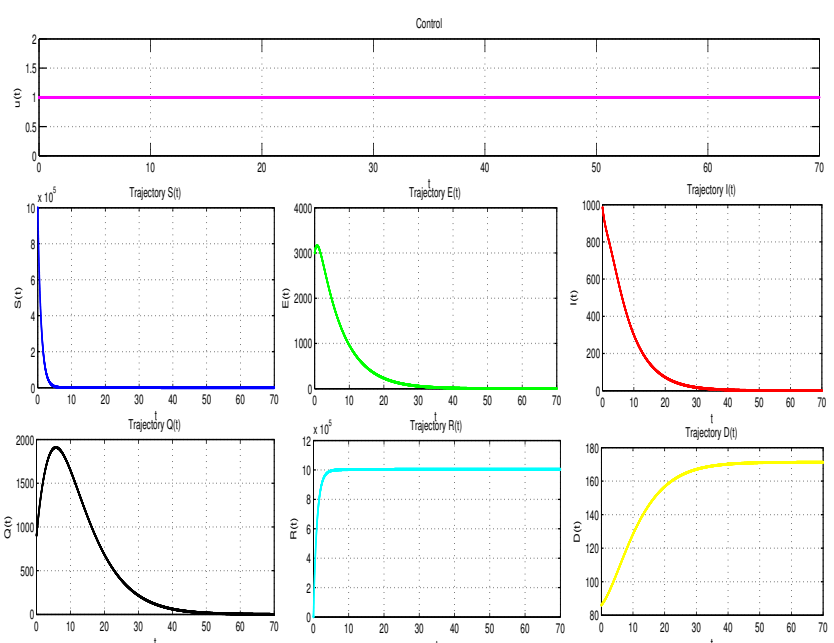

Figure 7. Evolution of stats with vaccine, after 70 days.

\section{Numerical comparison with results discussion}

The direct method is characterized by the simplicity of its implementation, without a priori knowledge of the solution, this method is not very sensitive to the choice of the initial condition, but it has low numerical precision. The indirect method has very high digital precision, but it is very sensitive to the choice of the initial condition.

We tried by this work and with two methods, to evaluate the considered states (in particular the infected and the recovered) with and without effective vaccine against Coronavirus. The results found by those methods are similar, but at least, the results found by shooting method are more accurate and the execution time of the shooting method is much less.

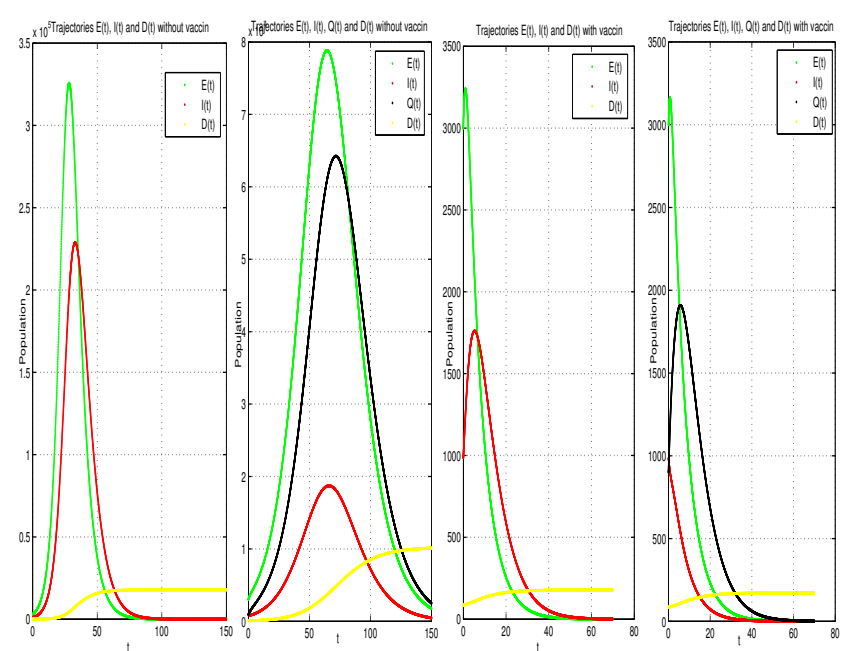

Figure 8. Numerical comparaison.

\section{Conclusion}

In this work, we have modeled a topical problem (COVID-19 problem) as a nonlinear optimal control problem. We considered the SEIR model by adding a compartment $D$ for dead persons. In the first part of the work, we added compartment $D$, then we discussed and compared the results, in the second part of the work, we included in the considered model, compartment $Q$ of people under quarantine.

First, the Pontryagin's maximum principle has been used which gives a necessary condition of optimality. By implementing two numerical methods (the shooting method and the Euler discretization method) on the MATLAB software, we have traced the evolutions of the states considered in the problem, at a duration of 70 days and 150 days with and without vaccine.

The results found show that the shooting method is fast and gives accurate results. We deduce that 
finding an effective vaccine is more than essential to fight against this virus. if not, the solution is quarantining the infected (see Figure 8).

\section{References}

[1] Kermack, W.O., \& McKendrick, A.G. (1927). Contributions to the mathematical theory of epidemics, part i. Proceedings of the Royal Society of Edinburgh. Section A. Mathematics, 115, 700-721.

[2] Goncalves, J.N.C., Rodrigues, H.S., \& Monteiro, M.T.T. (2018). On the dynamics of a viral marketing model with optimal control using indirect and direct methods. Statistics, Optimization $\&$ Information Computing, 6, 633-644.

[3] Rosa, S., \& Torres, D.F.M. (2018). Parameter Estimation, Sensitivity Analysis and Optimal Control of a Periodic Epidemic Model with Application to HRSV in Florida. Statistics, Optimization 8 Information Computing, 6, 139-149.

[4] Trélat, E. (2005). Optimal control: theory and applications. Vuibert, Concrete mathematics collection, Paris.

[5] Moussouni, N., \& Aidene, M. (2011). An Algorithm for Optimization of Cereal Output. Acta Applicandae Mathematicae, 11, 113-127.
[6] Moussouni, N., \& Aidene, M. (2016). Optimization of cereal output in presence of locusts. An International Journal of Optimization and Control: Theories \& Applications, 6, $1-10$.

[7] Aliane, M., Moussouni, N., \& Bentobache, M. (2020). Optimal control of a rectilinear motion of a rocket. Statistics, Optimization \&6 Information Computing, 8, 281-295.

[8] Aliane, M., Moussouni, N., \& Bentobache, M. (2019). Nonlinear optimal control of the heel angle of a rocket. 6th International Conference on Control, Decision and Information Technologies (CODIT'19), Paris, 756-760.

[9] Pontryagin, L.S., Boltyanskii, V.G., Gamkrelidze, R.V., \& Mishchenko, E.F. (1962). The mathematical theory of optimal processes. Intersciences Publisher, New York.

Nacima Moussouni received her PhD in Mathematics at the University of Tizi-Ouzou, Algeria. She is an Associate Professor at the University of Laghouat, Algeria and the university of Orlean in France(MAPMO laboratory). Her research interests include optimal control and its applications.

\section{(10) https://orcid.org/0000-0002-3570-2462}

Mohamed Aliane received his Master Degree in Analysis and Mathematical Modelling at the University of Medea, Algeria. He is a PhD student at the Department of Mathematics, University of Laghouat, Algeria. His research interests include optimal control and its applications.

(10)https://orcid.org/0000-0002-8020-4649

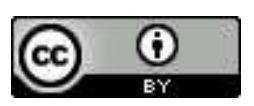

This work is licensed under a Creative Commons Attribution 4.0 International License. The authors retain ownership of the copyright for their article, but they allow anyone to download, reuse, reprint, modify, distribute, and/or copy articles in IJOCTA, so long as the original authors and source are credited. To see the complete license contents, please visit http://creativecommons.org/licenses/by/4.0/. 